Original Research

\title{
The Effect Of Mild Exercise Induced Dehydration On Sport Concussion Assessment Tool 3 (SCAT3) Scores: A within-subjects design.
}

\author{
Sean M Collins, $\mathrm{PhD}^{1}$ a , Monica R Lininger, PhD, ATC ${ }^{2}$, Thomas G Bowman, PhD, ATC \\ 1 Exercise Physiology Department, University of Lynchburg, ${ }^{2}$ Athletic Training Program, Northern Arizona University, ${ }^{3}$ Athletic Training Department, \\ University of Lynchburg \\ Keywords: movement system, concussion management, cognition, balance
}

https://doi.org/10.26603/001c.21534

\section{International Journal of Sports Physical Therapy}

Vol. 16, Issue 2, 2021

\section{Background}

Sports-related concussions are prevalent in the United States. Various diagnostic tools are utilized in order to monitor deviations from baseline in memory, reaction time, symptoms, and balance. Evidence indicates that dehydration may also alter the results of diagnostic tests.

\section{Purpose}

The purpose was to determine the effect of exercise-induced dehydration on performance related to concussion examination tools.

\section{Study Design}

Repeated measures design.

\section{Methods}

Seventeen recreationally competitive, non-concussed participants (age: $23.1 \pm 3.1$ years, height: $168.93 \pm 10.71 \mathrm{~cm}$, mass: $66.16 \pm 6.91 \mathrm{~kg}$ ) performed three thermoneutral, counterbalanced sessions (rested control, euhydrated, dehydrated). Participants were either restricted $(0.0 \mathrm{~L} / \mathrm{hr})$ or provided fluids $(1.0 \mathrm{~L} / \mathrm{hr})$ while treadmill running for $60 \mathrm{~min}$ at an intensity equal to 65-70\% age-predicted maximum heart rate (APMHR). The Sport Concussion Assessment Tool 3 (SCAT3) was utilized to assess symptoms, memory, balance, and coordination.

\section{Results}

Statistically significant differences were seen among sessions for symptom severity and symptom total. The rested control session had significantly lower values when compared to the dehydrated session. Additionally, the symptom total in the rested control was significantly lower than the euhydrated condition as well. No statistically significant differences were seen for the BESS or memory scores.

\section{Conclusions}

Mild exercise-induced dehydration results in increased self-reported symptoms associated with concussions. Clinicians tasked with monitoring and accurately diagnosing head trauma should take factors such as hydration status into account when assessing patients for concussion with the SCAT3. Clinicians should proceed with caution and not assume concussion as primary cause for symptom change.

\footnotetext{
Corresponding Author: 


\section{Level of evidence}

Level 3

\section{INTRODUCTION}

Sports-related concussions and the proper care of athletes with concussion symptoms is a growing concern in the healthcare field. Epidemiological analyses of sports-related concussions in the United States estimate there are between 1.6 and 3.8 million incidents per year. ${ }^{1}$ While cognitive deficits as a result of a single concussive event may not result in long-term impairments, repetitive concussions or a subsequent concussion prior to adequate recovery has been reported to lead to longer lasting effects. ${ }^{2,3}$ Therefore, properly identifying concussed patients and removing them from participation is critical to long term brain health. Further, due to the risks and high incidence rate of concussion, proper diagnosis is vital for proper administration of care and improved return to play timelines for athletes. Concussion detection tools utilize deviations in various symptoms, including short- and long-term memory, reaction time, and postural stability, from a baseline to determine likelihood of a concussive event. ${ }^{4,5}$ However, the nature of sport and physical activity may produce extraneous conditions that may result in similar symptoms or conditions that may disrupt the ability of a health care professional to accurately detect and manage patients with a concussion. ${ }^{6-9}$

Mild dehydration ( $<2 \%$ loss in $\mathrm{BM}$ ) has also been reported to alter variables examined to determine concussion. ${ }^{10-12}$ Landers, Arent, and Lutz ${ }^{13}$ reported non-significant cognitive disturbances amongst various tests for wrestlers who voluntarily dehydrated themselves for competition weight, whereas Lieberman et al. ${ }^{14}$ reported significant changes in reaction time, memory, attention, and reasoning in response to exercise-induced dehydration in a hyperthermic environment. These results indicate that exercise-induced dehydration, especially in the heat, may hinder cognitive performance. Similarly, Patel and colleagues $^{15}$ reported significant changes in visual memory, fatigue, and severity of symptom (headaches, dizziness, feeling slowed down, difficulty remembering, and difficulty concentrating) scores amongst collegiate wrestlers following bouts of exercise-induced dehydration. Additionally, Weber and colleagues ${ }^{8}$ also found deviations in the Balance Error Scoring System (BESS) and the Graded Symptoms Checklist (GSK) following bouts of weight cutting techniques and wrestling practice amongst collegiate wrestlers.

On-field concussion tests are conducted on the field of play under conditions where exercise-induced dehydration may play a role in influencing the diagnosis. The suggested on-field concussion examination tool, the Sport Concussion Assessment Tool 3 (SCAT3), utilizes the common detection methods of concussion: attention, memory, balance and reaction time. ${ }^{4}$ The detection tools need to be able to determine the likelihood of a concussion to allow proper management. Ideally, concussion examination techniques will be both sensitive and specific allowing practitioners to be confident in clinical decisions. Therefore, the purpose of this study was to determine the effect of exercise-induced dehydration on cognitive performance as detected by the
SCAT3.

\section{METHODS}

The current study used a counterbalanced repeated-measures designed to investigate the effect of exercise-induced dehydration on neuropsychological performance and postural stability as detected by the SCAT3.

\section{PARTICIPANTS}

For the experiment, a total of 17 recreationally competitive volunteers consisting of seven males (25.0 $\pm 3.7 \mathrm{yrs}$; $176.77 \pm 5.12 \mathrm{~cm} ; 68.62 \pm 3.92 \mathrm{~kg})$ and 10 females $(21.8 \pm 1.9 \mathrm{yrs}$; $162.82 \pm 9.98 \mathrm{~cm} ; 64.38 \pm 8.16 \mathrm{~kg}$ ), who regularly participated in 60 minutes of aerobic exercise three to four days per week, were recruited. The participants were excluded if they reported history of previously diagnosed concussion, heatrelated illness, back and neck injury, cardiovascular disease, vestibular dysfunction, history of ADHD or ADD, dyslexia and other learning disabilities, or any lower extremity injury that would inhibit balance. All participants were required to read and sign a PAR-Q, a Medical History Questionnaire, as well as read and sign an Informed Consent Form approved by the Institutional Review Board at the University of Lynchburg prior to participation.

\section{PROTOCOL}

Participants completed three counterbalanced testing sessions separated by at least $72 \mathrm{hrs}$. Each subject was required to wear shorts, a shirt, socks, undergarments, and shoes during each training session and were asked to wear the same garments for each subsequent testing session. Participants were expected to come hydrated but were given no guidance on diet and they were not asked to track their diet via a journal.

Prior to attending the first session to get a baseline score, the participants were required to refrain from strenuous exercise, alcohol, and caffeine for 24 hours. Upon arrival to the laboratory and completion of the PAR-Q and Informed Consent Form, participants provided a 60- to 120$\mathrm{mL}$ urine sample to determine urine specific gravity (USG, Palm Abbe 202X, Misco, Cleveland, OH). Anthropometric measurements including nude mass were determined via an electronic floor scale (BWB-800S, Tanita, Tokyo, Japan), a standing stadiometer (Seca, Hamburg, Germany), and body composition estimations via a Siri three-site skinfold measurement using handheld calipers (Lange, Cambridge, MA). Participants were excluded from testing if their USG was greater than or equal to 1.020 , which would classify them as already dehydrated.

Participants performed two exercise sessions and a baseline session in counterbalanced order. The baseline (control) session consisted of participants resting and consuming fluid ad libitum for $60 \mathrm{~min}$ prior to completing the data collection protocol. Each exercise session was performed 
Table 1: SCAT 3 scores stratified across sessions.

\begin{tabular}{|c|c|c|c|c|}
\hline & \multicolumn{4}{|c|}{ Mean \pm SD } \\
\hline & $\begin{array}{c}\text { Control } \\
(\mathrm{n}=16)\end{array}$ & $\begin{array}{c}\text { Euhydrated } \\
(\mathrm{n}=17)\end{array}$ & $\begin{array}{c}\text { Dehydrated } \\
(\mathrm{n}=16)\end{array}$ & $\begin{array}{c}\text { Total } \\
(\mathrm{N}=49)\end{array}$ \\
\hline Symptom Total & $1.31 \pm 1.45$ & $2.18 \pm 2.33$ & $3.94 \pm 3.79$ & $2.47 \pm 2.85$ \\
\hline Symptom Severity Total & $1.56 \pm 1.86$ & $3.24 \pm 4.04$ & $6.31 \pm 7.78$ & $3.69 \pm 5.41$ \\
\hline Memory Total & $28.31 \pm 1.25$ & $27.71 \pm 2.23$ & $27.31 \pm 2.02$ & $27.78 \pm 1.90$ \\
\hline BESS Total & $2.13 \pm 2.42$ & $2.88 \pm 3.12$ & $4.50 \pm 2.90$ & $3.16 \pm 2.95$ \\
\hline
\end{tabular}

on a motorized treadmill (Full Vision TMX425 Trackmaster, Drive Newton, KS) at an incline of $1.0 \%$ grade and a speed that elicited an average of $65 \%$ to $70 \%$ of age-predicted maximum heart rate (APMHR) as measured by an attached heart rate monitor chest strap and watch (validity = 0.97-1.0; Polar WearLink ${ }^{\circledR} 31$ transmiter and Polar RS400, Kempele, Finland) ${ }^{16}$ for 60 minutes.

Prior to each testing sessions, participants provided a 60 - to $120 \mathrm{~mL}$ urine sample and performed a nude weight. In randomized order, participants performed the aforementioned exercise protocol in a thermoneutral environment with restricted water intake (dehydrated) and required fluid consumption of $1 \mathrm{~L}$, via four $250 \mathrm{ml}$ doses every $15 \mathrm{~min}$ (euhydrated). After the 60-minute exercise protocol, participants performed a five-minute cool down at self-selected pace. After the cool down, participants provided a 60- to 120- mL urine sample and had their body mass measured after toweling dry. Hydration status post-test was determined as the percent change of body mass pre-test to posttest.

Following baseline hydration measurement, participants completed the SCAT3. The SCAT3 is the recommended onfield concussion assessment tool ${ }^{13}$ and contains a symptom checklist, the Standardized Assessment of Concussion (SAC), and a modified version of the Balance Error Scoring System (BESS). Scoring is the sum total of scores in each test within the SCAT3. Symptom checklist is scored as both presence of the symptom, providing a total number of symptoms score (max. score: 22 ) and severity score, summing the magnitude of each symptom (max. score: 132). The higher the score (maximum of 132) the more symptomatic the participant. The SAC was a cognitive assessment monitoring Immediate Memory (max score: 5), Concentration (max score: 15), Orientation (max score 5), and Delayed Recall (max score: 5 ) for a total of 30 points possible when summed together. The lower the score the more affected the memory of the participant. The modified BESS counts total errors (max score: 30 ) for each 20-sec eye closed conditions: double leg stance, single leg stance (non-dominant), and tandem stance on a firm surface. Lower scores represent better performance.

\section{STATISTICAL ANALYSIS}

Descriptive statistics were calculated for each the dependent variables (symptom score, symptom severity, modified BESS, and SAC total). To determine if there were differences across sessions (control, euhydrated, dehydrated), a one- way Repeated Measures Analysis of Variance was used for each of the four dependent variables. First, assumption testing of linearity, normality, and sphericity were assessed. If a violation in sphericity was found, a Greenhouse-Geisser adjustment was made. For statistically significant results from the RM ANOVA, pairwise post-hoc testing was completed. Data were analyzed using Statistical Package for the Social Sciences (IBM SPSS, Inc., 26.0, Chicago, IL, USA). An alpha level of $\leqslant 0.05$ was used for all analyses.

\section{RESULTS}

Descriptive statistics for the four dependent variables can be found in Table 1 . There was a statistically significant difference among the sessions for both the SCAT3 symptom severity scores $\left(F_{2,20}=4.61, p=0.034\right)$ and the SCAT3 symptom total $\left(F_{2,28}=5.64, p=0.009\right)$. The rested control session had a significantly lower mean symptom severity score $(1.56 \pm 1.86)$ than the dehydrated session $(6.31 \pm 7.78, p=$ $0.032)$. The rested control session had a significantly lower mean symptom score $(1.31 \pm 1.45)$ than the dehydrated session (3.94 $\pm 3.79, p=0.019)$. Additionally, the rested control session was significantly lower for symptom total when compared to the euhydrated session $(2.18 \pm 2.33, p=0.019)$. No statistically significant differences were seen for modified BESS or SAC total.

\section{DISCUSSION}

Best practices suggest that health care professionals (athletic trainers [ATs], physical therapists [PTs], and medical doctors [MDs]) examining patients suspected of sustaining a concussion use a multifaceted approach. In order to accurately diagnose a concussion, ATs focus on a number of functions, including reaction time, short- and long- term memory, balance, and self-reported physical symptoms. Deviations in these scores, from baseline, are used to indicate the possibility of a concussion. Zimmer et $\mathrm{al}^{17}$ reported that average baseline scores in the SCAT2 were $91.08 \pm 5.60$ with average number of symptoms reported at 1.75 and the SAC and BESS scores were 27.17 \pm 2.10 and $25.64 \pm 4.07$, respectively. Putukian et al. ${ }^{18}$ indicated significant correlations between SCAT2 scores and various individual patient outcomes with as little as a 3.5-point drop from baseline.

However, there are other conditions that can also result in alterations in these variables that may lead to incorrect diagnosis on the field using commonly used examination 
tools, like the SCAT3. Previous researchers have reported changes in memory, reaction time, and balance as a result of dehydration ${ }^{13,15,19}$ due to possible reduced neural capacity and negative impact due to reduced blood volume. ${ }^{20}$ Comparatively, the current project resulted in similar variations in symptoms commonly associated with concussions, but not balance or neurocognitive function. The researchers suspect a combination of dehydration and fatigue were the underlying factors that altered the participants' symptoms as compared to their control levels. Fatigue has previously been found to increase injury risk while decreasing performance. ${ }^{21,22}$ In addition, lower cerebral performance has been linked to an increased risk of non-contact ACL injury ${ }^{23}$ perhaps due to loss of central control. ${ }^{24}$ The researchers concede that dehydration and/or fatigue can make the SCAT3 more conservative by increasing false-positive diagnoses, but believe this is less concerning compared to falsenegative diagnoses where concussed patients would be allowed to inappropriately return to play and risk a variety of complications. In other words, it is more reasonable to hold a patient out of activity if a concussion cannot be ruled out regardless of whether it can be ruled in or not. However, effort should be made to provide the most accurate diagnoses possible during activity to improve clinical care.

Balance Scores: Mild exercise-induced dehydration resulted in higher (worse) modified BESS scores $(4.50 \pm 2.90)$ compared to control and euhydrated sessions, however, this difference was not statistically significant. Similarly, Patel and colleagues ${ }^{15}$ reported no deviation in the BESS scores with dehydration levels greater than those reported in this project $(2.50 \pm 0.63 \%$ vs $1.61 \pm 1.0 \%$, respectively). They speculated that a learning effect could have been the reason for the lack of significant differences between the control and experimental groups. It is unlikely a learning effect played a role in the current study due to the randomized order of sessions, indicating that the level of exercise intensity, exercise type, nor the dehydration level were significant enough to illicit an alteration in balance. The change we documented between the control group $(2.13 \pm 2.42)$ and the euhydrated group $(2.88 \pm 3.12)$ were much smaller than the difference between collegiate aged concussed (5.46 \pm 5.44) patients and their baseline test results $(3.87 \pm 3.99)$. Others have determined that dehydration alone has not been found to alter BESS scores (full version, not modified) in isolation; however, exercise combined with dehydration did alter BESS scores. ${ }^{8}$ It has been suspected that the level of dehydration experienced by the participants in the current study may be similar to those seen in clinical practice on the sidelines, indicating the importance of considering hydration levels during clinical sideline testing, but more testing is needed. ${ }^{15}$ Similarly, completing components of the SCAT in a different environment ${ }^{25-27}$ or after fatiguing exercise $^{22}$ compared to baseline has also been found to alter test scores. However, resting for 20 minutes after exercise negates the regression in BESS scores. ${ }^{28}$ It remains unknown if a rest period with or without fluid access after dehydrating exercise would bring modified BESS scores back to baseline.

Symptom Scores: Participants' self-reported symptom scores and symptom severity scores increased as a result of dehydration following fluid restricted exercise (Figure 1).

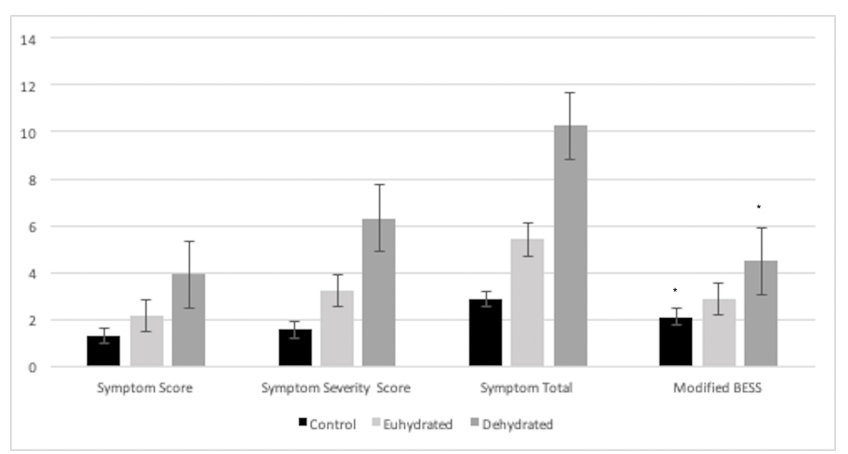

Figure 1: Mean score for symptoms and modified BESS testing by session.

*There was a significant difference between scores in the control session $(2.27 \pm$ $4.43)$ compared to the dehydrated session $(4.33 \pm 2.92)$.

The observed high standard deviations, similar to previous studies, strengthening the case for obtaining a baseline. ${ }^{8,15}$ A symptom severity increase was observed from the control $(1.56 \pm 1.86)$ to the euhydrated condition $(3.24$ \pm 4.04 ), albeit much smaller when compared to the difference between the dehydrated condition $(6.31 \pm 7.78)$, and not likely to be clinically significant. This pattern was also seen for symptom count in the sample with the lowest number of symptoms in the control session $(1.31 \pm 1.45)$, then in the euhydrated session $(2.18 \pm 2.33)$ and finally the highest number of symptoms in the dehydrated session (3.94 \pm 3.79). Previous studies have found elevated headache, feeling in a fog, and feeling slowed down symptom severity scores after dehydration. ${ }^{15}$ The reported symptom scores of the dehydrated group do fall within one standard deviation of reported symptom scores of concussed high school and collegiate athletes, ${ }^{29}$ indicating that there may a possible relationship between the physiological effects of dehydration and clinical symptoms of a concussion. Symptom severity scores may require multiple baseline measurements or measurements in different climates to improve clinical decisions, especially if more severe climate conditions are present when concussion is suspected, such as extreme heat and humidity which may exacerbate dehydration.

SAC Total: The effect of exercise and hydration status for SAC scores was parallel to the findings for balance. with no significant impact on SAC scores. Compared to the control session, the euhydrated sessions only scored an average of 0.6 points worse, compared to the dehydrated sessions 1.00-point degradation. Previous researchers have shown high sensitivity and specificity with a 1.00 -point difference between baseline and post-concussion assessments, ${ }^{29,30}$ which may suggest the findings of mean score differences after dehydrating exercise would be less indicative of a concussive event. Similarly, previous authors have shown no difference in SAC scores based on hydration status. ${ }^{8,15}$ In addition, other studies have found the SAC to be resistant to score changes based on the environment.6,27 Therefore, the SAC component to the SCAT3 may be more resistant to extraneous variables compared to balance and symptom scores.

Limitations and Future Directions: The data was collected 
from a convenience sample of seventeen recreationally active volunteers with various sport experiences. These participants may or may not respond to exercise similarly to professional, collegiate, or scholastic athletes. It would be interesting to examine athletes from different sports, fitness levels, and levels of acclimatization to heat and/or humidity to determine how dehydration may alter balance, symptoms, and mental status. In addition, the exercise protocol required 60 minutes of steady state running on a treadmill which is an activity uncommon in most sports and shorter than the duration of most athletic practices and games. Future research could determine whether more interval type exercise protocols for a period of time closer to many practices ( 2 hours) with and without fluid restriction would make more or less of an effect on SCAT3 scores. Finally, monitoring diet would have also brought more insight to the effect of exercise and dehydration on performance in field concussion testing.

\section{CONCLUSION}

The present findings indicate that worsened symptom and symptom severity scores occurred in subjects during the fluid restricted exercise condition compared to those exercising with unlimited fluids and a control session, in healthy, non-concussed subjects. As such, clinicians working with athletes and tasked with monitoring and accurately diagnosing head trauma should take factors such as hydration status into account when assessing patients for concussion using the SCAT3. If dehydration is suspected, patients should be provided time to rehydrate if necessary to ensure accurate diagnosis.

\section{CONFLICTS OF INTEREST}

No conflicts of interest to report by all authors.

Submitted: April 02, 2020 CDT, Accepted: October 10, 2020

CDT 


\section{REFERENCES}

1. LaRoche AA, Nelson LD, Connelly PK, Walter KD, McCrea MA. Sports-related concussion reporting and state legislative effects. Clin J Sport Med. 2016;26(1):33-39. doi:10.1097/jsm.000000000000019 $\underline{2}$

2. Guskiewicz KM, McCrea M, Marshall SW, et al. Cumulative effects associated with recurrent concussion in collegiate football players: The NCAA Concussion Study. JAMA. 2003;290(19):2549-2555. do i:10.1001/jama.290.19.2549

3. Randolph C, Millis S, Barr WB, et al. Concussion symptom inventory: An empirically derived scale for monitoring resolution of symptoms following sportrelated concussion. Arch Clin Neuropsych. 2009;24(3):219-229. doi:10.1093/arclin/acp025

4. Broglio SP, Cantu RC, Gioia GA, et al. National Athletic Trainers' Association position statement: management of sport concussion. J Athl Train. 2014;49(2):245-265. doi:10.4085/1062-6050-49.1.07

5. McCrory P, Meeuwisse WH, Aubry M, et al. Consensus statement on concussion in sport. $\mathrm{Br} \mathrm{J}$ Sports Med. 2013;47(5):250-258. doi:10.1136/bjsport s-2013-092313

6. Onate JA, Beck BC, Van Lunen BL. On-field testing environment and balance error scoring system performance during preseason screening of healthy collegiate baseball players. J Athl Train.

2007;42(4):446-451.

7. Schneiders AG, Sullivan SJ, Kvarnström J, Olsson M, Ydén T, Marshall S. The effect of footwear and sportssurface on dynamic neurological screening for sportrelated concussion. J Sci Med Sport. 2010;13(4):382-386. doi:10.1016/j.jsams.2010.01.003

8. Weber AF, Mihalik JP, Register-Mihalik JK, Mays S, Prentice WE, Guskiewicz KM. Dehydration and performance on clinical concussion measures in collegiate wrestlers. J Athl Train. 2013;48(2):153-160. doi:10.4085/1062-6050-48.1.07

9. Vaughan CG, Gerst EH, Sady MD, Newman JB, Gioia $\mathrm{GA}$. The relation between testing environment and baseline performance in child and adolescent concussion assessment. Am J Sports Med. 2014;42(7):1716-1723. doi:10.1177/036354651453173 2
10. Sell TC, Clark NC, Wood D, Abt JP, Lovalekar M, Lephart SM. Single-leg balance impairments persist in fully operational military special forces operators with previous history of low back pain. Ortho J Sport Med. 2014;2(5):1-6. doi:10.1177/2325967114532780

11. Tomporowski PD, Beasman K, Ganio MS, Cureton $\mathrm{K}$. Effects of dehydration and fluid ingestion on cognition. Int J Sports Med. 2007;28(10):891-896. do $\mathrm{i}: 10.1055 / \mathrm{s}-2007-965004$

12. Wilson M-MG, Morley JE. Impaired cognitive function and mental performance in mild dehydration. Eur J Clin Nutr. 2003;57(S2):S24-S29. do i:10.1038/sj.ejen.1601898

13. Landers DM, Arent SM, Lutz RS. Affect and cognitive performance in high school wrestlers undergoing rapid weight loss. J Sport Exerc Psych. 2001;23(4):307-316. doi:10.1123/jsep.23.4.307

14. Lieberman HR, Bathalon GP, Falco CM, Kramer FM, Morgan CA III, Niro P. Severe decrements in cognition function and mood induced by sleep loss, heat, dehydration, and undernutrition during simulated combat. Bio Psychiatry. 2005;57(4):422-429. doi:10.1016/j.biopsych.2004.11.014

15. Patel AV, Mihalik JP, Notebaert AJ, Guskiewicz KM, Prentice WE. Neuropsychological performance, postural stability, and symptoms after dehydration. $J$ Ath Train. 2007;42(1):66-75.

16. Engström E, Ottosson E, Wohlfart B, Grundström $\mathrm{N}$, Wisén $\mathrm{A}$. Comparison of heart rate measured by Polar RS400 and ECG, validity and repeatability. $A d v$ Physiother. 2012;14(3):115-122. doi:10.3109/1403819 6.2012.694118

17. Zimmer A, Marcinak J, Hibyan S, Webbe F. Normative values of Major SCAT2 and SCAT3 components for a college athlete population. App Neuropsychol Adult. 2015;22(2):132-140. doi:10.1080/ $\underline{23279095.2013 .867265}$

18. Putukian M, Echemendia R, DettwilerDanspeckgruber A, et al. Prospective clinical assessment using sideline concussion assessment tool-2 testing in the evaluation of sport-related concussion in college athletes. Clin J Sport Med. 2015;25(1):36-42. doi:10.1097/jsm.000000000000010 $\underline{2}$ 
19. Cian C, Barraud PA, Melin B, Raphel C. Effects of fluid ingestion on cognitive function after heat stress or exercise-induced dehydration. Inter J Psychophys. 2001;42(3):243-251. doi:10.1016/s0167-8760(01)0014 $\underline{2-8}$

20. Benton D, Young HA. Do small differences in hydration status affect mood and mental performance? Nutr Rev. 2015;73(suppl 2):83-96. doi:1 $\underline{0.1093 / \text { nutrit/nuv045 }}$

21. Cortes N, Greska E, Kollock R, Ambegaonkar J, Onate JA. Changes in lower extremity biomechanics due to a short-term fatigue protocol. J Athl Train. 2013;48(3):306-313. doi:10.4085/1062-6050-48.2.03

22. Wilkins JC, McLeod TC, Perrin DH, Gansneder BM. Performance on the balance error scoring system decreases after fatigue. J Athl Train. 2004;39(2):156-161.

23. Swanik CB, Covassin T, Stearne DJ, Schatz P. The relationship between neurocognitive function and noncontact anterior cruciate ligament injuries. Am J Sports Med. 2007;35(6):943-948. doi:10.1177/0363546 507299532

24. McLean SG, Samorezov JE. Fatigue-induced ACL injury risk stems from a degradation in central control. Med Sci Sport Exerc. 2009;41(8):1661-1672. $\underline{\mathrm{d}}$ oi:10.1249/mss.0b013e31819ca07b
25. Onate JA, Guskiewicz KM, Riemann BL, Prentice WE. A Comparison of sideline versus clinical cognitive test performance in collegiate athletes. $J$ Athl Train. 2000;35(2):155-160.

26. Rahn C, Munkasy BA, Joyner AB, Buckley TA. Sideline performance of the balance error scoring system during a live sporting event. Clin J Sport Med. 2015;25(3):248-253. doi:10.1097/jsm.0000000000000 141

27. Smith DH, Bowman TG, Bradney DA. The effect of the environment on sport concussion assessment tool 2 (SCAT2) scores. Athl Train Sports Health Care. 2016;8(5):193-201. doi:10.3928/19425864-2016051 $\underline{0-01}$

28. Susco TM, McLeod TC, Gansneder BM, Shultz SJ. Balance recovers within 20 minutes after exertion as measured by the Balance Error Scoring System. J Athl Train. 2004;39(3):241-246.

29. Chin EY, Nelson LD, Barr WB, McCrory P, McCrea MA. Reliability and validity of the Sport Concussion Assessment Tool-3 (SCAT3) in high school and collegiate athletes. Am J Sport Med. 2016;44(9):2276-2285. doi:10.1177/036354651664814 1

30. McCrea M. Standardized mental status testing on the sideline after sport-related concussion. J Athl Train. 2001;36(3):274-279. 